\title{
РАСШИРЕНИЕ ЧАСТОТНОГО
}

\section{ДИАПАЗОНА ВЫСОКОМОЩНЫХ ДЕЛИТЕЛЕЙ-СУММАТОРОВ МОЩНОСТИ}

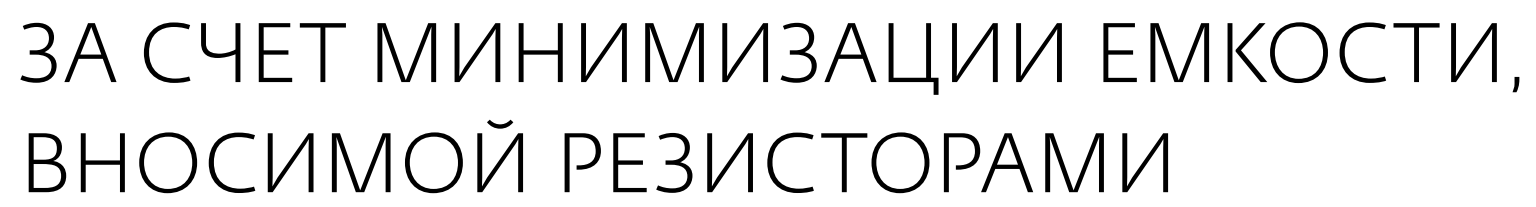

\section{А.Лезинов ${ }^{1}$}

УДК 621.372 .62 BAK 05.27 .00

\begin{abstract}
ZACS242-100W+ - мощный синфазный делитель-сумматор мощности с коаксиальными разъемами на два выхода с допустимой мощностью на входе в режиме делителя до 100 Вт, разработанный компанией Mini-Circuits. Высокая допустимая мощность на входе этого устройства позволяет использовать его в мощных системах распределения сигналов, работающих в диапазоне частот от 500 до 2400 МГц. Однако на частотах выше 2400 МГц вносимые потери устройства начинают возрастать, ограничивая его применение в высокочастотных изделиях. Рассмотрим, как можно изменить конструкцию данного делителя, чтобы расширить полосу пропускания на 50\% за счет снижения потерь сигнала из-за влияния паразитных емкостей, вносимых используемыми в устройстве чипрезисторами. Предлагаемый авторами подход был реализован в новой модели делителя-сумматора мощности - ZACS362-100W+, который отличается низкими вносимыми потерями на частотах вплоть до 3600 МГц.
\end{abstract}

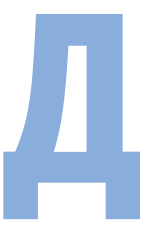
опустимая мощность на входе делителей определяется, в сущности, номинальной мощностью внутренних резисторов, которая связана с их размерами - чем выше мощность, тем крупнее резистор. В делителях, рассчитанных на входную мощность 100 Вт, применяются достаточно габаритные резисторы, поэтому важно учитывать влияние связанных с ними паразитных емкостей на вносимые потери.

В делителе ZACS242-100W+ используются четыре 100-Вт чип-резистора (рис.1). Емкость между металлизи-

АО "ЮЕ-Интернейшнл", руководитель группы ВЧ-продукции, alexey.lezinov@yeint.ru. рованными контактными поверхностями на нижней части каждого резистора и печатной платой можно выразить соотношением для емкости между двумя параллельными обкладками конденсатора:

$$
C=\frac{\varepsilon_{\mathrm{a}} A}{\mathrm{~d}}
$$

где $\varepsilon_{\text {a }}$-абсолютная диэлектрическая проницаемость материала между двумя обкладками;

A - площадь перекрытия обкладок;

$\mathrm{d}$ - расстояние между обкладками.

Другими словами, если принять постоянными значения $\varepsilon_{\text {a }}$ и d, то чем больше площадь проводящей поверхности на нижней части резистора, 


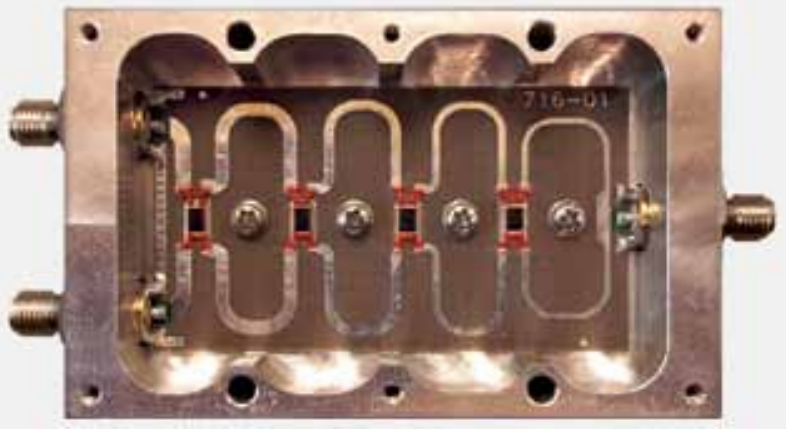

Рис.1. Печатная плата делителя-сумматора мощности ZACS242-100W+ с четырьмя 100-Вт чип-резисторами

перекрывающей площадку на печатной плате, тем выше паразитная емкость, связанная с данным резистором. Эта емкость (рис.1) увеличивает суммарные вносимые потери схемы, особенно на высоких частотах, что в основном и ограничивает полосу пропускания делителя ZACS242-100W+.

Целью изменения конструкции было расширение частотного диапазона делителя ZACS242-100W+ при обеспечении низкого уровня вносимых потерь и сравнимых общих характеристик схемы с характеристиками исходной конструкции. Один из путей решения задачи-снижение паразитной емкости, вносимой чип-резисторами. Отметим, что резисторы в делители ZACS242-100W+ установлены перевернутыми лицевой стороной вниз; при этом все проводящие контактные поверхности расположены параллельно печатной плате. В данном случае невозможно использовать резисторы меньших размеров или уменьшить площадь контактов, однако, чтобы минимизировать площадь перекрытия проводящих плоскостей, можно иначе расположить чип-резистор на печатной плате.

Поэтому в конструкцию устройства были внесены изменения - чип-резисторы были повернуты на $90^{\circ}$ относительно поверхности печатной платы (рис.2). Такое расположение резисторов приводит к уменьшению вносимой ими паразитной емкости более чем в десять раз, что, в свою очередь, значительно снижает общие вносимые потери на высоких частотах.

Измерения вносимых потерь при свипировании частоты подтвердили улучшение данной характеристики в новых делителях ZACS362-100W+ с вертикально установленными чип-резисторами по сравнению с предыдущей моделью - ZACS242-100W+, в которой резисторы расположены параллельно печатной плате (рис.3).

В то время как у делителя ZACS242-100W+ вносимые потери возрастают на частотах выше 2400 МГц, в модифицированной версии-ZACS362-100W+-достигаются низкие значения вносимых потерь вплоть до частоты 3600 МГц, что эквивалентно 50\%-ному расширению рабочей полосы частот. Обе модели обеспечивают допустимую мощность на входе на уровне 100 Вт в режиме делителя. Однако, если ZACS242-100W+ в режиме сумматора допускает входную мощность $40 \mathrm{BT}$, то для новой модели -ZACS362-100W+- этот показатель равен 5 ВТ. В остальном ZACS362-100W+ имеет сравнимые с ZACS242-100W+ характеристики вплоть до частоты 3600 МГц, при этом обеспечивается высокое значение переходного затухания (типовое значение 22 дБ) и малая асимметрия фазы и амплитуды (1 и 0,15 дБ, соответственно).

$$
\because *
$$

Конструкторское решение, представленное в данной статье, основано на учете емкости, создаваемой
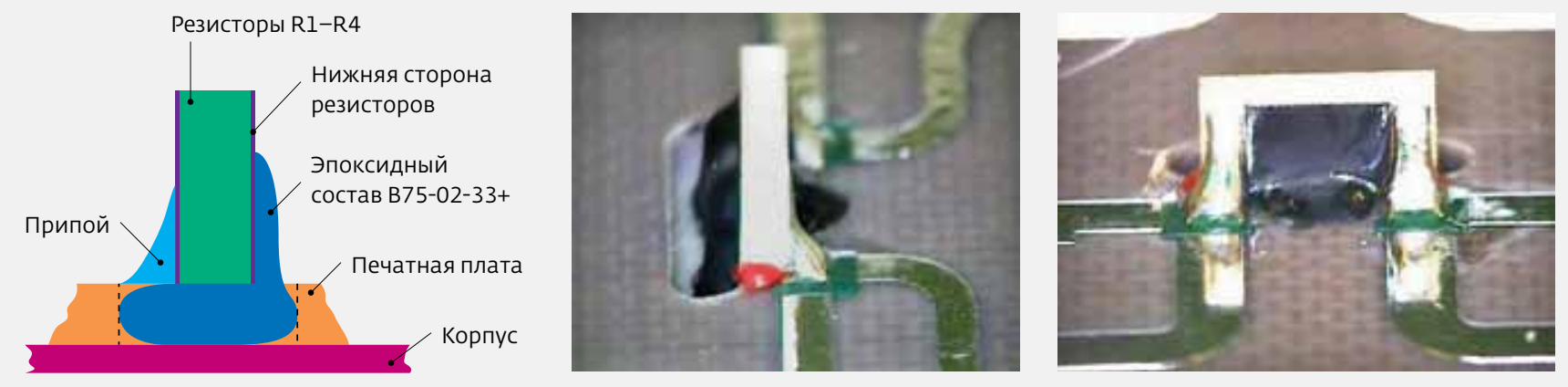

Рис.2. Чип-резисторы расположены так, что их проводящие поверхности перпендикулярны поверхности печатной платы 

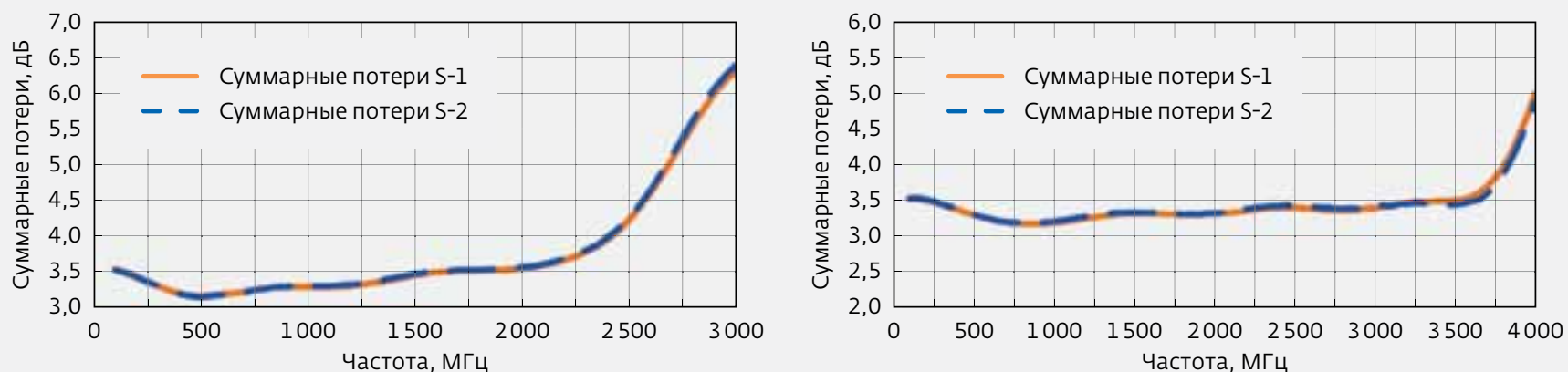

Рис.3. Сравнение частотных зависимостей вносимых потерь для исходной (слева) и новой (справа) конструкций делителя-сумматора мощности

параллельными плоскостями, и направлено на минимизацию паразитной емкости, вносимой чип-резисторами, в схеме делителя-сумматора мощности. В результате его реализации удалось значительно снизить вносимые потери на высоких частотах. Этот же подход был использован для расширения частотного диапазона до значений, достигающих 6 ГГц, в других моделях мощных делителей-сумматоров в продуктовой линейке компании
Mini-Circuits. В настоящее время компания разрабатывает новые модели устройств с аналогичной величиной допустимой мощности, рабочая частота которых достигает 12 ГГц.

Материалы предоставлены АО "ЮЕ-Интернейшнл" (www.yeint.ru), официальным дистрибьютором компании Mini-Circuits в России.

\section{КНИГИ ИЗДАТЕЛЬСТВА "ТЕХНОСФЕРА"}

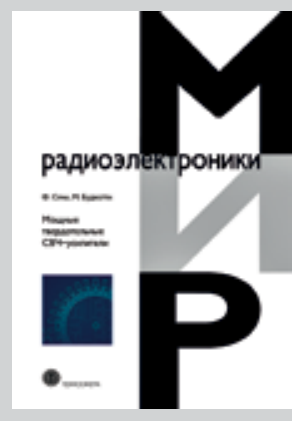

Цена 975 руб.
МОЩНЫЕ ТВЕРДОТЕЛЬНЫЕ СВЧ-УСИЛИТЕЛИ Сечи Ф., Буджатти М.

При поддержке АО "НПП «Исток» им. Шокина" Перевод с англ. nод ред. д.т.н. А.А.Борисова

В книге рассмотрены все традиционные вопросы, связанные с разработкой усилителей мощности, начиная от получения моделей приборов на большом сигнале и заканчивая обсуждением сумматоров мощности и методов проектирования.

Большое внимание в издании уделено рассмотрению физических основ приборов, фазовых шумов, схем смещения и тепловому проектированию. Также в книге особое внимание уделяется рассмотрению фундаментальных принципов. Издание затрагивает необычайно большое количество областей, связанных с физикой полупроводников и активных устройств.

Книга представляет интерес для специалистов, которые занимаются разработкой усилителей мощности для базовых станций сотовой связи. В особенности это относится к рассмотрению моделей на больших сигналах, проблем, связанных с фазовыми шумами, методов проектирования усилителей мощности, специальных конструкций усилителей мощности и теплового проектирования. Также данная книга может послужить в качестве справочного пособия при углубленном изучении СВЧ-устройств.

КАК ЗАКАЗАТЬ НАШИ КНИГИ?

$凶$ 125319, Москва, а/я 91; \(495) 234-0110; 皿 (495) 956-3346; knigi@technosphera.ru, sales@technosphera.ru 